\title{
Modelling the effects of release timing on the wind- assisted dispersal of passive propagules
}

\author{
David Savage $^{\mathrm{a}, \mathrm{b}}$, Martin J. Barbettia,b, William J. MacLeod ${ }^{\mathrm{a}, \mathrm{b}, \mathrm{c}}$, Moin U. Salam ${ }^{\mathrm{b}, \mathrm{c}}$ and Michael \\ Renton $^{\mathbf{a}, \mathbf{b}, \mathbf{d}, \mathbf{e}}$ \\ ${ }^{1}$ School of Plant Biology and Institute of Agriculture, University of Western Australia, WA, Australia \\ ${ }^{2}$ Cooperative Research Centre for National Plant Biosecurity, ACT, Australia \\ ${ }^{3}$ Department of Agriculture and Food Western Australia, WA, Australia \\ ${ }^{4}$ CSIRO Ecosystem Sciences, WA, Australia \\ ${ }^{5}$ Centre of Excellence for Climate Change, Forest and Woodland Health, WA, Australia \\ Email:david.savage@mac.com
}

\begin{abstract}
Wind-assisted dispersal operates across a range of scales, facilitating the interaction of meteorological, biological and physical processes acting at the source location, across the path of dispersing individuals and at their eventual destination. For passively dispersing propagules such as seeds, pollen and fungal spores, the wind-conditions experienced over the course of their journey largely define the distance and direction of their dispersal. For a given location, temporal patterns in the release of propagules can interact with prevailing wind conditions at both diurnal and seasonal scales. This paper presents the results from a series of investigations into the effects of these interactions, examining the influence of release timing on wind-assisted dispersal, and the development of methods for integrating knowledge of release timing into simple models of biological spread.

In two previous studies, we used existing well-validated models of wind-assisted dispersal to investigate the effect of seasonal and diurnal patterns of propagule release on wind-assisted dispersal. Results of these studies showed that the major direction of spread, and the distance and area covered by an individual dispersal event is influenced by release timing. In particular, both the hour of the day, and the season in which propagules are released were shown to influence the probability that long-distance dispersal will occur. In addition to these studies, we have also previously developed a simple method for constructing mechanistically parameterised dispersal kernels, which are commonly used to model dispersal in a computationally efficient manner. Our method takes into account the wind-conditions that occur at a given location over a given period of time, and allows kernels to be parameterised using only meteorological data, and the physical characteristics of the organism in question. The resulting kernels are two-dimensional and can represent the anisotropic nature of wind-assisted dispersal, which results from the non-uniform distribution of wind directions at a given location. Comparison of kernels with existing models of windassisted dispersal has shown that our method is capable of generating a realistic representation of windassisted dispersal. In this paper, we demonstrate that kernels constructed using our method are also able incorporate prior knowledge of temporal release patterns, and show that the inclusion of temporal release patterns in kernel construction can result in considerable differences to similarly constructed kernels that assume no pattern of release. We argue that our method of kernel construction can enable more accurate modelling of specific dispersal events, resulting spread and (meta-)population dynamics. This will be of major benefit in areas such as biosecurity, conservation ecology, human health and integrated pest management, where greater understanding of the movement of pathogens, or endangered species, can lead to significant benefits.
\end{abstract}

Keywords: Diurnal, Dispersal kernel, Release timing, Wind-assisted dispersal 


\section{INTRODUCTION}

Wind-assisted dispersal is an important ecological process that commonly occurs in many plants, fungi, and some small invertebrates (Edmonds, 1979, Nathan et al., 2005). Depending on the organism in question, wind-assisted dispersal can occur across a range of scales, and can facilitate the interaction of genetic, physiological, and meteorological factors between spatially separated locations. The environment, genetic makeup and life history of a particular individual can all effect its likelihood for successful dispersal, and, in turn, the success or failure of large numbers of individuals to disperse between particular locations affects the the spatial structure and temporal dynamics of (meta-)populations and communities, and the evolution of related traits.

The specific timing of propagule release facilitates interaction between the factors that condition a propagule for release, and those that define a propagule's transport and deposition (Soons and Bullock, 2008, Kuparinen et al., 2009, Savage et al., 2011a). In particular, the timing of release determines the wind conditions encountered by a dispersing propagule over the course of its journey, and these conditions almost completely define the distance and direction over which dispersal occurs. Periods of high wind speeds or high degrees of turbulent flow can result in propagules experiencing sustained upward drafts, enabling them to disperse over much larger distances than would normally be possible (Soons et al., 2004, Kuparinen et al. 2009, Savage et al., 2011a). Over many individual dispersal events, the timing of release will affect the direction, distance and area of spread for the population(s) in question. Therefore, timing of release plays an important role in determining the invasive capabilities of an organism, the ability of (meta-)populations to survive in a given environment, the ability of separated populations to exchange genetic material, and the speed at which novel, or newly selected for alleles are spread through a population. These phenomenon are often modelled in significant detail in order to provide realistic predictions of biological spread and (meta-)population dynamics for use in disciplines such as biosecurity (Coutts et al., 2011), pest management (Diggle et al., 2002, Skelsey et al., 2009), and conservation ecology (Soons et al., 2004, Bohrer et al., 2005). Within these disciplines, understanding the role of release timing in the dispersal of propagules can enable practitioners to more accurately model specific dispersal events and specific organisms in specific environments, allowing the effects of management strategies to be tested in a more robust manner. Therefore, the purpose of this paper is to synthesise a number of results stemming from our research into the timing of propagule release, and to draw some general conclusions and recommendations relating to the modelling of wind-assisted dispersal. In particular, we describe a method for incorporating knowledge of release timing into simple models of wind-assisted dispersal, and demonstrate the improvements that this method provides over those that do not take the timing of release into account.

\section{EFFECTS OF TEMPORAL PATTERNS OF PROPAGULE RELEASE ON WIND-ASSISTED DISPERSAL}

Wind conditions change over hourly and seasonal time scales. Many regions experience prevailing conditions (e.g. an afternoon sea breeze) that can be assumed to occur with some regularity. However, even strongly prevailing winds are subject to variation, and wind conditions at the same location can be expected to vary significantly over a suitable period of time. Consequently, small changes in the timing of release can lead to large differences in the direction, distance and area covered by dispersal stemming from a particular release event (Savage et al., 2010, Savage et al., 2011a).

Long distance dispersal is a particularly important aspect of wind-assisted dispersal (and dispersal in general), allowing invasive organisms to colonise new areas (Isard et al., 2007), to cross hostile, uninhabitable regions, and to survive as meta-populations in fragmented environments (Bohrer et al., 2005). The occurrence of long-distance wind-assisted dispersal is greatly increased by particular meteorological conditions that promote high degrees of turbulent updrafts (Soons et al., 2004, Kuparinen et al., 2009, Savage et al., 2011a). Such conditions are characterised by high horizontal wind speeds, and also high sensible heat flux, which describes the transfer of heat energy from the earth's surface into the atmosphere (Soons et al., 2004). Both of these meteorological variables follow seasonal and diurnal patterns, resulting in interactions between meteorological conditions and seasonal and diurnal patterns of propagule release (Kuparinen et al., 2009, Savage et al., 2011a). In particular, investigations at Merredin and Mt Barker in Western Australia have shown that horizontal wind speed and sensible heat flux peak during the mid afternoon, and that release of fungal spores at this time is more likely to result in long-distance dispersal than release occurring at any other time of the day (Savage et al., 2011a). Similarly, release at these locations during September resulted in a higher proportion of spores undergoing long-distance dispersal than release in June due to the occurrence of warmer temperatures and the resulting higher sensible heat flux (Savage et al., 2011a). 


\section{INTEGRATING PATTERNS OF PROPAGULE RELEASE INTO MODELS OF WIND- ASSISTED DISPERSAL}

In modelling the wind-assisted dispersal of small propagules, phenomenological models have been used with success in a number of studies (e.g. Diggle et al., 2002, Bohrer et al., 2005, Coutts et al., 2011). Phenomenological models describe the observed pattern of dispersal, but ignore the underlying processes that generate this pattern. Where these models are fitted to real data, they implicitly take into account the effects of release timing on dispersal, however, constructing realistic phenomenological models for a particular organism at a particular time in a particular location can be challenging, as data is required to be collected over large areas and relatively long periods of time (Katul et al., 2005, Nathan et al., 2005). Therefore, more mechanistic approaches have become popular, which can be more easily parameterised to represent a particular situation. This includes the mechanistic parameterisation of dispersal kernels (e.g. Tufto et al., 1997, Katul et al., 2005, Savage et al., 2011c), and the use of more traditional mechanistic models such as Lagrangian stochastic models (e.g. Aylor and Flesch, 2001, Soons et al., 2004), Eulerian density models (e.g. Savage et al., 2010, Savage et al. 2011c) or Gaussian plume or puff models (e.g. Skelsey et al., 2009). While these models continue to be improved, the possibility of integrating patterns of release has not been widely examined (but see Schippers and Jongejans, 2005, Soons and Bullock, 2008). However, as previously shown (Kuparinen et al., 2009, Savage et al., 2010, Savage et al., 2011a), patterns of release can play an important role in determining the conditions experienced by a propagule over the course of it's journey, and by integrating descriptions of release patterns, these models can be greatly improved.

\subsection{Quantifying and describing temporal patterns of propagule release}

In order to integrate temporal patterns of release into existing models of wind-assisted dispersal, we clearly need a description of the temporal pattern for the organism of interest. However, obtaining such a description can pose a significant challenge, as different mechanisms of release exist across the broad range of winddispersing organisms, and within a given organism, patterns of release may vary over time or between different locations depending on the environmental conditions experienced. In some organisms, the process of release is not well understood, and many of the environmental variables that drive release have not yet been identified (e.g. Inch et al., 2005, Savage et al., 2011b). Therefore, characterisation of release patterns in these organisms has to date relied on phenomenological, rather than mechanistic, descriptions.

In describing the challenges associated with characterising temporal patterns of propagule release, we consider the fungal pathogen Leptosphaeria maculans as an example, as this pathogen exhibits a number of attributes that make the characterisation of temporal release patterns difficult. L. maculans is an important agricultural pest that causes blackleg disease of oilseed Brassica crops. The pathogen is widespread globally, and is responsible for significant economic losses in oilseed production areas. Ascospores released by $L$. maculans undergo wind-assisted dispersal and are believed to be capable of dispersal over at least tens of kilometres (West and Fitt, 2005). Seasonal patterns of ascospore release (i.e days consisting of release events) depend on rainfall, which is required to trigger release, and also the accumulated temperature, which influences the maturation of ascospores within the pathogen's fruiting bodies (Salam et al., 2003). Diurnal patterns of release have also been observed (Huang et al., 2005, Savage et al., 2011b), however these patterns differ depending on the location and time of observation (Savage et al., 2011b). At present, the mechanistic process of ascospore release is not well understood, and while some meteorological variables such as rainfall, humidity and temperature have been correlated with release (Huang et al., 2005, Salam et al., 2003, Savage et al., 2011b), these are not sufficient to make accurate predictions of both seasonal and diurnal patterns of release. Moreover, observations of a Western Australian L. maculans population over three consecutive years showed that diurnal patterns of release can vary between different years, and between different months within the same year (Savage et al., 2011b). Diurnal patterns exhibited by this population also differed from those observed in England and Canada, and from those observed for the related L. biglobosa in Poland. These results demonstrate that phenomenological descriptions of diurnal and seasonal patterns should not be extrapolated to different geographic regions, or to different periods of time without testing the validity of these models across a range of locations, and that it would be better if mechanistic descriptions of temporal release patterns could be employed. However, for areas of application such as biosecurity, predictions of wind-assisted dispersal are required to test the effects of management policies prior to the arrival of the organism in question. Therefore, where mechanistic descriptions of temporal release patterns are not available, extrapolation of some sort will be required, and modelling should be performed using a wide range of possible patterns of release as part of a full risk analysis.

\subsection{Filtering input data to account for temporal patterns of propagule release}

Integrating temporal patterns of release into existing forms of dispersal models can be achieved in two ways. Firstly, the process of release can be explicitly represented as a sub-component of a dispersal model, or secondly, the inputs provided to a dispersal model can be modified and filtered to reflect the specific patterns 
of release for the particular location, time period, and organism being modelled. In this paper, we concentrate on the second method, which has the added advantage of requiring no changes to be made to existing model codes.

As an example of how input to an existing model can be modified to account for temporal patterns of release, consider the commonly used Lagrangian stochastic form of dispersal model (e.g. Aylor and Flesch, 2001, Soons et al., 2004, Schippers and Jongejans, 2005, Kuparinen et al. 2009, Savage et al., 2011a). These models generally take an average horizontal wind speed as input, and may also accept other, temporally averaged, meteorological variables. Averaging of these meteorological variables can easily be performed for appropriate time periods, giving, for example, seasonal or diurnal patterns for these variables (e.g. Savage et al., 2011a). Given a pattern of release for a particular organism, the proportion of propagules dispersed during a given time period can be calculated, and the appropriate values for meteorological variables can then be used to drive the simulation. In this way a more detailed description of dispersal is achieved, with any effects of temporal variation on wind conditions accounted for in the overall results.

While Lagrangian stochastic models can provide a highly realistic description of wind-assisted dispersal, their computational requirements makes them unsuitable for use in larger scale simulations of (meta-)population dynamics or biological invasions over large time scales and large geographic regions. More commonly, dispersal kernels are used in these situations (e.g. Diggle et al., 2002, Bohrer et al., 2005, 2010, Coutts et al., 2011), which describe the probability that an individual propagule will disperse over a given distance. Dispersal kernels have traditionally been fitted to data collected through trapping of airborne propagules, or direct observation of successful dispersal, which attempt to measure the distances travelled by dispersing individuals. Often such data is difficult to collect, and has therefore limited the use of dispersal kernels where modelling requires dispersal to be characterised at a particular location, for a particular period of time (Katul et al., 2005, Nathan et al., 2005, Savage et al., 2011c). The use of some forms of fitted dispersal kernels has also been criticised for a lack of biological relevance of the resulting parameter set (Tufto et al., 1997). However, these problems can be largely overcome through the use of mechanistically parameterised dispersal kernels, which consist of biologically relevant parameters that can be estimated from the meteorological and biological characteristics of the situation being modelled, and therefore, do not require the collection of difficult to obtain dispersal data (Tufto et al., 1997, Katul et al., 2005, Savage et al., 2011c).

A recent study showed that mechanistically parameterised kernels can be constructed to represent the twodimensional, anisotropic dispersal for a specific organism, location, and period of time (Savage et al., 2011c). These dispersal kernels describe the probability of dispersal into a given two-dimensional area using a joint probability distribution consisting of a half Cauchy or inverse Gaussian distribution, which describes the probability of dispersal over a given distance, and a von Mises distribution, which describes the probability of dispersal in a given direction. For the purposes of this paper, we use the method for kernel construction outlined in this study to demonstrate the manner in which data can be filtered to obtain a two-dimensional, anisotropic dispersal kernel that accounts for temporal patterns in propagule release. The constructed kernel consists of a half Cauchy distribution, with the shape parameter $\gamma$ taken as the median distance travelled, obtained using

$$
\gamma=\frac{h u}{v_{t}}
$$

where $h$ is the height of propagule release, $u$ is the mean horizontal wind speed for the location and time period of interest, and $v_{t}$ is the terminal velocity of the propagule being modelled. Note that in this formulation of the model, each of the variables $h, u$ and $v_{t}$ could also be taken as distributions if the level of variation in the scenario being modelled warranted this approach.

The effects of temporal patterns of release on wind assisted dispersal can be included in the mechanistically parameterised dispersal kernel in much the same way as for the Lagrangian stochastic model. By appropriately filtering the wind speed and direction data for the temporal pattern of interest, and by taking each of the kernel parameters as a stepwise function of time, we effectively generate $n$ kernels, where $n$ is the number of time periods in the temporal pattern of interest. These kernels can then be combined to form a mixture by taking the sum over all kernels, with each kernel's contribution to the mixture weighted by the proportion of the total spores released in the corresponding period. This mixture is defined as

$$
k=\sum_{i=1}^{n} a_{i} \cdot k_{i} \quad\left(a_{i}>0, \quad \sum_{i=1}^{n} a_{i}=1\right)
$$


where $k$ is the resulting kernel, $k_{i}$ is the kernel for the time period $i$, and $\alpha_{i}$ is the proportion of spores released in the time period $i$. As an example, if we are considering a diurnal pattern of release, we might generate 24 sub-kernels, representing average dispersal in each hour of the day. In calculating the overall dispersal kernel, the contribution of each sub-kernel to the mixture would be weighted by the proportion of spores released in the corresponding hour. Figure 1 shows an example of a diurnal pattern, which is the same as that observed in a Western Australian population of L. maculans over the period June - August, 1972 - 1974 (Savage et al., 2011b). This particular diurnal pattern shows a peak in release at $6 \mathrm{pm}$, and is expressed in terms of the proportion of spores released in a given hour. Therefore, if we multiply the proportion by the number of spores released on a given day, we would obtain the number of spores released in each hour.

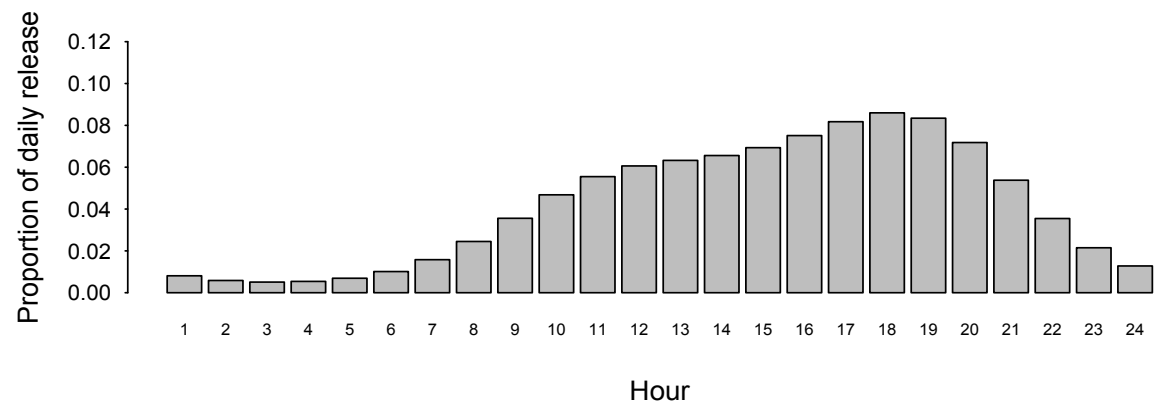

Figure 1: A diurnal pattern of release showing the probability that spore release will occur in a given hour. This pattern is same as that observed in a Western Australian population of Leptosphaeria maculans over the period June - August, 1972 - 1974 (Savage et al., 2011b).

Figure 2 shows the results of applying the method outlined in Savage et al. (2011a) to construct two dispersal kernels based on meteorological data for Mt Barker over the period May - August, 2000 - 2008. The constructed kernels assume a release height of $0.24 \mathrm{~m}$, and a terminal velocity of $0.001 \mathrm{~m} / \mathrm{s}$. Panel A shows a kernel that assumes no temporal pattern, while panel B takes into account both seasonal and diurnal patterns of release. For panel B, the meteorological data was first filtered to only include those hours where rainfall had occurred in the previous 12 hours, as rainfall has been previously shown to be an important factor in triggering release in L. maculans (Salam et al., 2003, Savage et al., 2011b). The filtered data was then split into 24 sub-sets based on the hour of the day, and used to construct 24 sub-kernels representing the expected dispersal for each hour. The overall dispersal kernel was then calculated using the hourly proportions of spores released for the diurnal pattern shown in Figure 1.
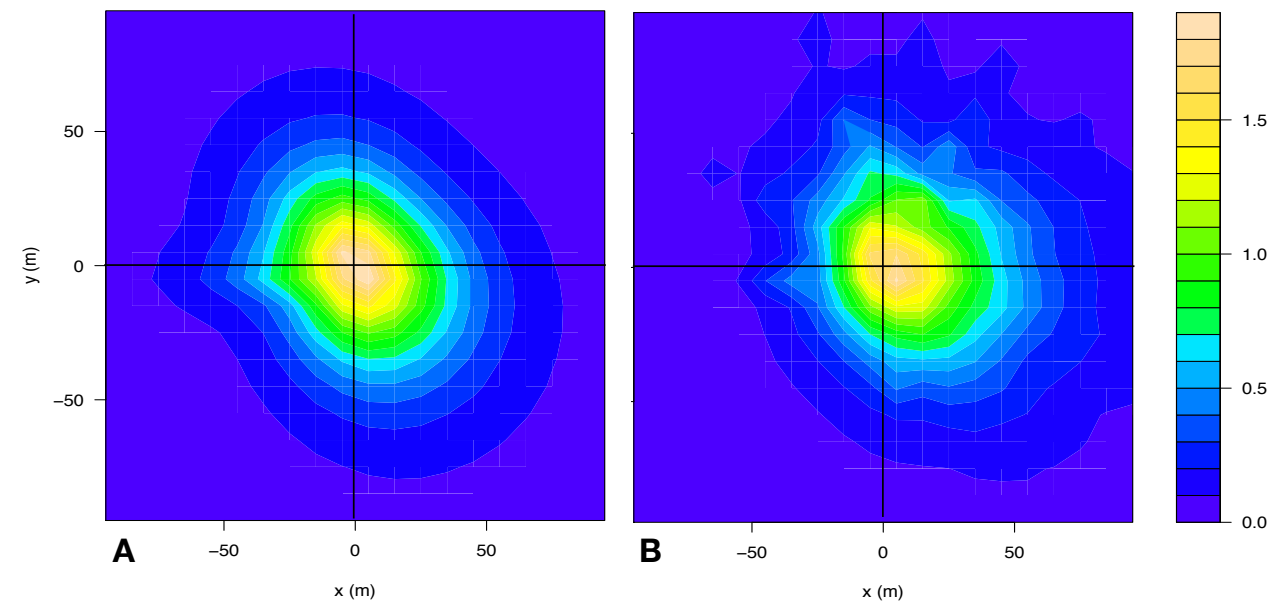

Figure 2: Two-dimensional anisotropic kernels constructed using meteorological data for Mt Barker over the period May - August, 2000 - 2008. The kernels show the effects of assuming no temporal pattern of

release (A), compared to filtering meteorological data to only include those hours where rainfall had occurred in the previous 12 hours, and weighting the contribution of date from each hour according to a diurnal pattern of release $(\mathbf{B})$. The density scale shows $\log (1+\log (1+$ spore count $))$, with the total number of spores released set to 10,000 .

Comparison between Panels A and B in Figure 2 shows that the inclusion of temporal patterns of release results in a greater probability for dispersal to the East and North-East, with propagules expected to travel both further and more frequently in these directions but expected to travel smaller distances and less 
frequently towards the West. While the differences between the kernels may seem relatively small, we would expect that over an extended period of time, and numerous dispersal events, the spread of a population and the resulting spatial distribution would be significantly different for each of the kernels shown and that the differences between them would be significantly magnified. This can be seen in Figure 3 , which shows the results of simulations using the two kernels shown in Figure 2. This simulation was performed using the General Model of Biological Invasion (Renton et al. 2011), and consists of a starting population of ten mature individuals. In each time step mature individuals produce ten offspring, which disperse to a random location, determined by sampling the two-dimensional distribution described by the dispersal kernel. Once they have dispersed, individuals mature, and produce offspring until they experience mortality, which occurs with a probability of $p=0.2$.

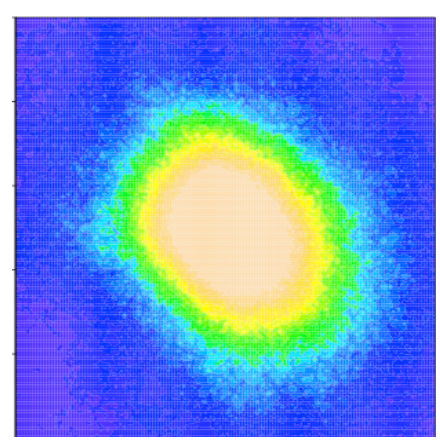

A

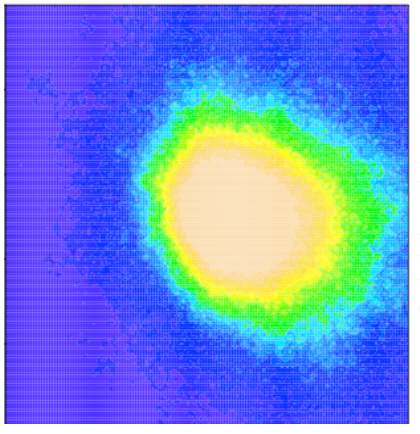

B

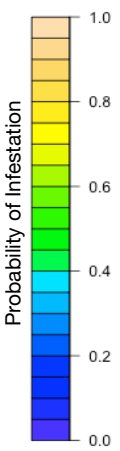

C

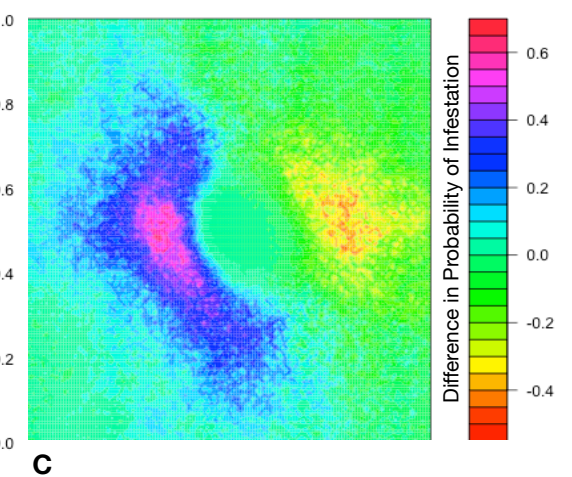

Figure 3: The probability that a given cell will be infested over 100 simulations of biological spread resulting from a dispersal kernel with no temporal patterns taken into consideration (A), and with both seasonal and diurnal patterns taken into account (B). The area shown in each panel represents a region of $225 \mathrm{~km}^{2}$, and the density scale shows the (square root of the) probability that a given cell will be infested. At this scale, the small differences between the dispersal kernels can result in significant differences in the area of spread (C).

\section{CONCLUDING NOTES}

Temporal patterns of propagule release can significantly influence the process of wind-assisted dispersal. Both the direction and distance travelled by dispersing propagules are determined by the wind-conditions experienced over the course of a propagules journey, and these conditions are set by the timing of a propagules release. Since the the timing of release in some pathogens can vary across geographical regions and across different time periods, further efforts should be made to characterise temporal patterns of release, and to gain a mechanistic understanding of the process of release. Such understanding would allow temporal patterns of release to be simulated under changing environmental conditions. Where temporal patterns of release can be reasonably estimated, these patterns can be incorporated into existing models of wind-assisted dispersal through the filtering of input meteorological data to reflect the timing of release. As shown in this paper, the inclusion of temporal release patterns will allow more accurate modelling of specific dispersal events, and resulting spread and (meta-)population dynamics. This can be of major benefit in areas such as biosecurity, conservation ecology, human health and integrated pest management, where greater understanding of the movement of pathogens, or endangered species, can lead to improved management practices.

\section{ACKNOWLEDGEMENTS}

The authors acknowledge the support of the Cooperative Research Centre for National Plant Biosecurity, established and supported under the Australian Government's Cooperative Research Centres Programme. The authors would also like to acknowledge the support of the Department of Food and Agriculture Western Australia for contributing to the salary of Martin Barbetti.

\section{REFERENCES}

Aylor, D.E., and Flesch, T.K. (2001). Estimating spore release rates using a Lagrangian stochastic simulation model. Journal of Applied Meteorology, 40, 1196-1208.

Bohrer, G., Nathan, R., and Volis, S. (2005). Effects of long-distance dispersal for metapopulation survival and genetic structure at ecological time and spatial scales. Journal of Ecology, 93, 1029-1040. 
Coutts, S., Klinken, R., Yokomizo, H., and Buckley, Y. (2011). What are the key drivers of spread in invasive plants: Dispersal, demography or landscape: And how can we use this knowledge to aid management? Biological Invasions, 13, 1649-1661.

Diggle, A.J., Salam, M.U., Thomas, G.J., Yang, H.A., OConnell, M., and Sweetingham, M.W. (2002). Anthracnosetracer: A spatiotemporal model for simulating the spread of Anthracnose in a lupin field. Phytopathology, 92, 1110-1121.

Edmonds, R.L. (1979). Aerobiology: The Ecological Systems Approach, edited by Edmonds, R.L. (Dowden, Hutchinson \& Ross Inc., Stroudsburg, Pennsylvania, 1979), 1-10.

Huang, Y.J., Fitt, B.D.L., Jedryczka, M., and Dakowska, S. (2005). Patterns of ascospore release in relation to phoma stem canker epidemiology in England (Leptosphaeria maculans) and Poland (Leptosphaeria biglobosa). European Journal of Plant Pathology, 111, 263-277.

Inch, S., Fernando, W.G.D., and Gilbert, J. (2005). Seasonal and daily variation in the airborne concentration of Gibberella zeae (schw.) petch spores in manitoba. Canadian Journal of Plant Pathology, 27, 357-363.

Isard, S.A., Russo, J.M., and Ariatti, A. (2007). The integrated aerobiology modeling system applied to the spread of soybean rust into the Ohio river valley during September 2006. Aerobiologia, 23, 271-282.

Katul, G.G., Porporato, A., Nathan, R., and Siqueira, M. (2005). Mechanistic analytical models for longdistance seed dispersal by wind. American Naturalist, 166, 368-381.

Kuparinen, A., Katul, G.G., Nathan, R., and Schurr, F. (2009). Increases in air temperature can promote wind-driven dispersal and spread of plants. Proceedings of the Royal Society B: Biological Sciences, 276, 3081-3087.

Nathan, R., Sapir, N., Trakhtenbrot, A., and Katul, G.G. (2005). Long-distance biological transport processes through the air: Can nature's complexity be unfolded in silico. Diversity \& Distributions, 11, 131-137.

Paulitz, T.C. (1996). Diurnal release of ascospores by Gibberella zeae in inoculated wheat plots. Plant Disease, 80, 674-678.

Renton, M. Savage, D., Chopard, J. (2011) A general spatially-explicit model to inform rapid response to new biological invasions: why we need one and what it should look like? In Chan, F., D. Marinova and R. S. Anderssen (eds) MODSIM2011 International Conference on Modelling and Simulation. Forthcoming.

Salam, M.U., Khangura, R.K., Diggle, A.J., and Barbetti, M.J. (2003). Blackleg Sporacle: A model for predicting onset of pseudothecia maturity and seasonal ascospore showers in relation to blackleg of canola. Phytopathology, 93, 1073-1081.

Savage, D., Barbetti, M.J., MacLeod, W.J., Salam, M.U., and Renton, M. (2010). Timing of propagule release significantly alters the deposition area of resulting aerial dispersal. Diversity \& Distributions, 16, 288-299.

Savage, D., Barbetti, M.J., MacLeod, W.J., Salam, M.U., and Renton, M. (2011a). Seasonal and diurnal patterns of spore release affect the probability that long-distance wind-assisted dispersal will occur. Microbial Ecology (in press).

Savage, D., Barbetti, M.J., MacLeod, W.J., Salam, M.U., and Renton, M. (2011b). Temporal patterns of ascospore release in Leptosphaeria maculans vary depending on the time and location of observation. Phytopathology (under review).

Savage, D., Barbetti, M.J., MacLeod, W.J., Salam, M.U., and Renton, M. (2011c). Can mechanistically parameterised, anisotropic dispersal kernels provide a reliable estimate of wind-assisted dispersal? Ecological Modelling, 222, 1673-1682.

Schippers, P., and Jongejans, E. (2005). Release thresholds strongly determine the range of seed dispersal by wind. Ecological Modelling, 185, 93-103.

Skelsey, P., Kessel, G.J.T., Rossing, W.A.H., and Werf, W. v. d. (2009). Parameterization and evaluation of a spatiotemporal model of the potato late blight pathosystem. Phytopathology, 99, 290-300.

Soons, M.B., and Bullock, J.M. (2008). Non-random seed abscission, long-distance wind dispersal and plant migration rates. Journal of Ecology, 96, 581-590.

Soons, M.B., Heil, G.W., Nathan, R., and Katul, G.G. (2004). Determinants of long-distance dispersal by wind in grasslands. Ecology, 85, 3056-3068.

Tufto, J., Engen, S., and Hindar, K. (1997). Stochastic dispersal processes in plant populations. Theoretical Population Biology, 52, 16-26.

West, J.S., and Fitt, B.D.L. (2005). Population dynamics and dispersal of Leptosphaeria maculans (blackleg of canola). Australasian Plant Pathology, 34, 457-461. 